\title{
Entre Pensamiento y Acción. Carlos Varsavky y la puesta en marcha de la empresa ALUAR
}

\begin{abstract}
Resumen: Se analiza la perspectiva tecnológica inicial de la empresa ALUAR durante su puesta en marcha entre 1974 y 1976 y el papel de Carlos Varsavsky y su estrategia tecnológica, que permitió enfrentar los desafíos técnicos de la producción de aluminio primario en Argentina. En el marco del Pensamiento Latinoamericano en Ciencia, Tecnología y Desarrollo (PLACTED), se muestra cómo, en una estrecha relación entre pensamiento y acción, la propuesta pragmática de Varsavsky de combinar tecnología extranjera con recursos humanos altamente formados en el sistema científico argentino y un plan de desarrollo interno para modernizarla con los años permitió generar un estilo y sendero tecnológico propio en ALUAR. A pesar del temprano alejamiento de Varsavsky, las capacidades endógenas gestadas en ALUAR durante sus primeros años madurarían en la década siguiente en una serie de innovaciones y mejoras incrementales de gran envergadura.
\end{abstract}

Palabras claves: ALUAR; aluminio primario; PLACTED; estilo tecnológico; autonomía científica y tecnológica.

Between Thought and Action. Carlos Varsavky and the start-up of the ALUAR company

\begin{abstract}
The article analyzes the initial technological perspective of the ALUAR company during its start-up between 1974 and 1976 and the role of Carlos Varsavsky and his technological strategy, which allowed the technical challenges of primary aluminum production in Argentina. Within the framework of Latin American Thought in Science, Technology and Development (PLACTED), it is shown how, in a close relationship between thought and action, Varsavsky's pragmatic proposal to combine foreign technology with highly trained human resources in the Argentine scientific system and an internal development plan to modernize it over the years allowed ALUAR to generate its own style and technological path. Despite Varsavsky's early departure, the endogenous capabilities developed at ALUAR during its early years would mature in the following decade into a series of far-reaching incremental innovations and improvements.
\end{abstract}

Keywords: ALUAR; primary aluminum; PLACTED; technological style; scientific and technological autonomy.

Entre o pensamento e a ação. Carlos Varsavsky e o início da empresa ALUAR

Resumo: Analisaremos neste artigo a perspectiva tecnológica inicial da empresa ALUAR, durante seu início entre 1974 e 1976, bem como o papel de Carlos Varsavsky e sua estratégia tecnológica, que permitiu enfrentar os desafios técnicos da produção de alumínio primário na Argentina. No marco do Pensamento Latino-Americano em Ciência, Tecnologia e Desenvolvimento (PLACTED), mostra-se como, em estreita relação entre pensamento e ação a proposta pragmática de Varsavsky de combinar tecnologia estrangeira com recursos humanos altamente capacitados no sistema científico argentino possuindo igualmente um plano de desenvolvimento interno para modernizá-lo ao longo dos anos, permitiu a geração de um estilo e percurso tecnológico próprios na ALUAR. Apesar da partida precoce de Varsavsky, as capacidades endógenas desenvolvidas na ALUAR durante seus primeiros anos amadureceriam na década seguinte em uma série de inovações e melhorias incrementais de longo alcance.

Palavras-chave: ALUAR; alumínio primário; PLACTED; estilo tecnológico; autonomia científica e tecnológica.

\section{Ciencia Tecnología y Política Año $4 N^{\circ} 7$ Noviembre 2021}

María Noelia Corvalán

Carro

Magíster en Ciencia,

Tecnología e Innovación.

Grupo de Investigación en

Gestión, Desarrollo Territorial y Ambiente.

Universidad Tecnológica

Nacional-Regional Chubut.

mnoelia.corvalan@gmail.com

Andrés Niembro

Doctor en Economía.

Universidad Nacional de Río

Negro. Instituto de Estudios

en Ciencia, Tecnología,

Cultura y Desarrollo.

aniembro@unrn.edu.ar

Año $4 N^{\circ} 7$ Noviembre 2021

Fecha de recibido: 07/04/2021

Fecha de aprobado: 05/10/2021

https://doi.org/10.24215/26183188e062

https://revistas.unlp.edu.ar/CTyP ISSN 2618-3188

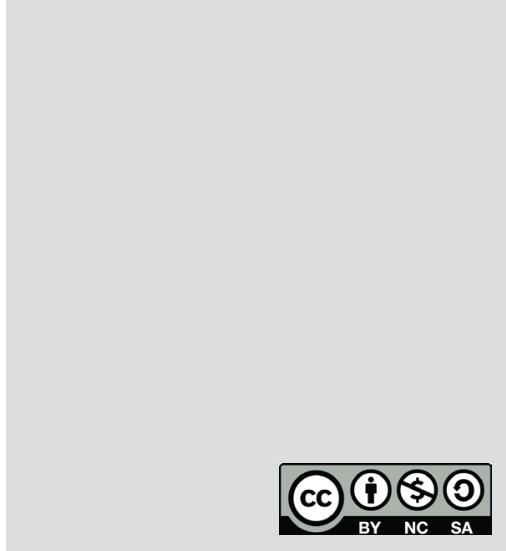

Esta obra está bajo licencia Creative Commons Atribución-NoComercial-Compartirlgual 4.0 Internacional http://creativecommons.org/licenses/bync-sa/4.O/deed.es_AR 


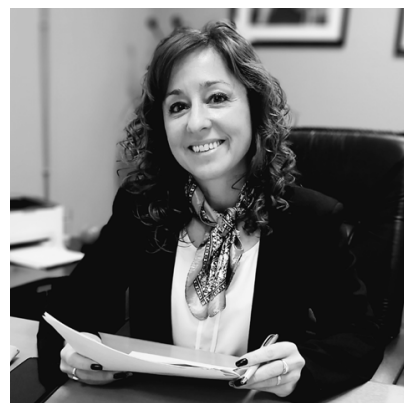

María Noelia Corvalán Carro

Magíster en Ciencia,

Tecnología e Innovación.

Grupo de Investigación en

Gestión, Desarrollo Territorial y Ambiente.

Universidad Tecnológica

Nacional-Regional Chubut.

mnoelia.corvalan@gmail.com

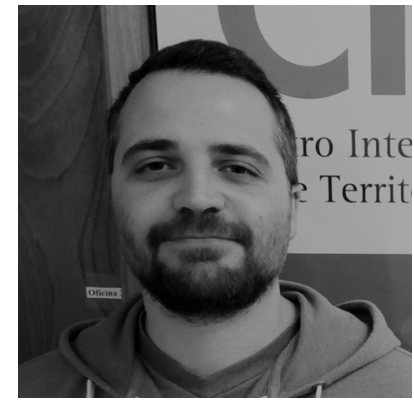

Andrés Niembro

Doctor en Economía.

Universidad Nacional de Río

Negro. Instituto de Estudios

en Ciencia, Tecnología,

Cultura y Desarrollo.

aniembro@unrn.edu.ar

\section{Entre pensamiento y acción. Carlos Varsavky y la puesta en marcha de la empresa ALUAR}

Resumen: Se analiza la perspectiva tecnológica inicial de la empresa ALUAR durante su puesta en marcha entre 1974 y 1976 y el papel de Carlos Varsavsky y su estrategia tecnológica, que permitió enfrentar los desafíos técnicos de la producción de aluminio primario en Argentina. En el marco del Pensamiento Latinoamericano en Ciencia, Tecnología y Desarrollo (PLACTED), se muestra cómo, en una estrecha relación entre pensamiento y acción, la propuesta pragmática de Varsavsky de combinar tecnología extranjera con recursos humanos altamente formados en el sistema científico argentino y un plan de desarrollo interno para modernizarla con los años permitió generar un estilo y sendero tecnológico propio en ALUAR. A pesar del temprano alejamiento de Varsavsky, las capacidades endógenas gestadas en ALUAR durante sus primeros años madurarían en la década siguiente en una serie de innovaciones y mejoras incrementales de gran envergadura.

\section{Introducción}

Durante las décadas del 60' y 70' se dio forma al denominado Pensamiento Latinoamericano en Ciencia, Tecnología y Desarrollo (PLACTED), en un contexto de crítica tanto al modelo de innovación lineal ofertista instaurado a partir de los años 50' como a las determinaciones tecnológicas que habían asumido los procesos de industrialización por sustitución de importaciones (ISI) en América Latina. Como destacan Galante y Marí (2020), este movimiento congregó a tecnólogos, científicos e intelectuales que sostenían la necesidad de desarrollar (endógenamente) una autonomía científica y tecnológica propia, y que además mostraron una "estrecha relación entre pensamiento y acción" (Sábato, 1972 , p. 7). En esta corriente pueden ubicarse (más allá de algunas diferencias de posturas entre si) autores como Amílcar Herrera, Jorge Sábato y Oscar Varsavsky en la Argentina, Helio Jaguaribe y José Leite Lopes en Brasil, Osvaldo Sunkel en Chile, Máximo Halty Carrere en Uruguay, Francisco Sagasti en Perú, Miguel Wionczek en México y Marcel Roche en Venezuela 
| Ciencia, Tecnología y Política | Año 4 | N7 | e062 | Noviembre 2021 | ISSN 2618-3188 | www.revistas.un|p.edu.ar/CTyP |

(Dagnino et al., 1996).

En esta misma etapa se gestó la industria del aluminio primario en Argentina, que al igual que otras contó con el amparo de regímenes promocionales de la segunda fase de la ISI. La génesis de esta industria debe entenderse en el marco de la estrategia nacional de desarrollo tecnológico e industrial orientada al autoabastecimiento de manufacturas, y particularmente de la autosuficiencia de este material estratégico para el complejo militar-industrial-estatal (Harriague et al., 2007; Rougier, 2011). Por otra parte, varios autores sostienen que el modelo sustitutivo había posibilitado la evolución de la industria del aluminio desde lo más sencillo hacia fases más complejas (Bisang, 1994; Rougier, 2011), pero el desarrollo del sector enfrentaba la ausencia del eslabón de producción de aluminio primario en el país. Esto representaba un factor de compleja resolución, por tratarse de una industria de rendimientos decrecientes que demandaba grandes escalas de producción.

En los intentos por expandir la cadena hacia el eslabón primario, se crea en 1966 la Comisión Permanente de Planeamiento para el Desarrollo de Metales Livianos (COPEDESMEL), dependiente de la Secretaría de Estado de Aeronáutica. Sus estudios y recomendaciones contribuyeron en la definición de la localización de la planta de producción de aluminio en Puerto Madryn, como lugar estratégico cercano a la fuente de provisión de energía y de un puerto de aguas profundas. Bajo el gobierno militar de Levingston (1970-71) y a pesar de los fuertes cuestionamientos de diferentes sectores involucrados y oferentes desestimados, se contrata directamente a ALUAR, creada en 1970 para el concurso público como estrategia de diversificación de la empresa FATE. Previamente a la firma del contrato, el llamado a licitación se había declarado desierto mediante el Decreto $N^{\circ} 206 / 71$, dado que ninguna de las tres propuestas presentadas cumplían con los requisitos técnicos, financieros y empresariales. Años más tarde, el 8 de agosto de 1974, se realiza la primera colada de aluminio totalmente producido en Argentina.

Las particularidades (o no tanto) de la historia hicieron que el apellido Varsavsky estuviera muy presente en los hechos hasta aquí relatados: Oscar, como un claro referente del PLACTED; y su primo Carlos, un reconocido científico y una de las figuras clave en el proyecto y puesta en marcha de ALUAR. Según De Alto (2012), "Oscar, era casi doce años mayor, por ello, y por su talento, era admirado por Carlos. Esa diferencia de edad no permitió una relación cómplice [en su juventud], pero sus respectivas y descollantes trayectorias merecen analizar paralelos y divergencias". Este artículo busca destacar el rol, la estrategia y las acciones de Carlos Varsavsky en los inicios de ALUAR y su relación con algunos de los preceptos centrales del PLACTED. ${ }^{1}$

\footnotetext{
${ }^{1}$ Carlos Varsavsky nació en Buenos Aires en 1933 y, tras cursar sus estudios secundarios en el Colegio Nacional de Buenos Aires, se radicó en los Estados Unidos con una beca para estudiar ingeniería física en la Universidad de Colorado. Luego, en 1959 completó su doctorado en astronomía en la Universidad de Harvard. Al año siguiente retornó a Argentina y se incorporó al grupo de astrofísica en la Universidad de Buenos Aires, donde se desempeñó como profesor titular hasta la fatídica "noche de los bastones largos" en 1966. Durante estos años también fue el primer director del Instituto Argentino de Radioastronomía (IAR), creado en 1962, donde se construyó el radiotelescopio más grande del hemisferio sur (bautizado en 2019 con el nombre de Carlos Varsavsky). Luego de la UBA y el IAR, vino la experiencia de la División Electrónica de FATE y la construcción y puesta en marcha de ALUAR. En 1977, tras el secuestro y desaparición de su sobrino por parte de la dictadura militar, emigró junto a su familia a los Estados Unidos. Wassily Leontief, premio Nobel de Economía, lo recibió como director asociado del Instituto de Análisis Económico de la Universidad de Nueva York, cargo que ocupó hasta su fallecimiento en 1983.
} 
Como se destaca al inicio del documento fundacional de la Red de Pensamiento Latinoamericano en Ciencia, Tecnología y Sociedad (Red PLACTS, 2020, p. 1), "uno de los desafíos históricos que enfrentan Argentina, en particular, y América Latina y el Caribe, en general, está vinculado con la integración de la ciencia y la tecnología al desarrollo nacional". Cuando se plantean objetivos propios, nacionales o regionales, "las tecnologías que nos ofrecen los países centrales no suelen dar respuesta a gran parte de los problemas prácticos que estos objetivos nos obligan a resolver y debemos tener una decidida actitud creativa (...) construir nuestro propio estilo científico-tecnológico (Red PLACTS, 2020, p. 5, parafraseando a Varsavsky, 1974). En esta línea, Oscar Varsavsky (1972, p. 88-89) señalaba que:

no se trata de rechazar en bloque la tecnología y la ciencia del Norte, sino de no aceptar todo en bloque (...): tener criterios propios de selección, prioridades para la asignación de recursos. Tampoco se trata sólo de adaptar tecnologías a nuestros recursos (...), aunque esto es indispensable. Se trata en primer lugar de aprender a usar objetivos nacionales, necesidades sociales, como criterios últimos para evaluar métodos de producción y tipos de organización y las tecnologías y ciencias que se requieren.

Varias de estas ideas se verán reflejadas en lo que sigue del artículo, donde se repasa, primero, el papel de algunos actores clave en la gestación del proyecto ALUAR y, particularmente, de Carlos Varsavsky y su estrategia tecnológica. Finalmente, se destacan algunos de los desafíos técnicos y la estructura organizacional de la firma en sus primeros años, cerrando con las conclusiones del caso.

\section{Actores claves del proyecto ALUAR}

Si bien el proyecto de producción nacional de

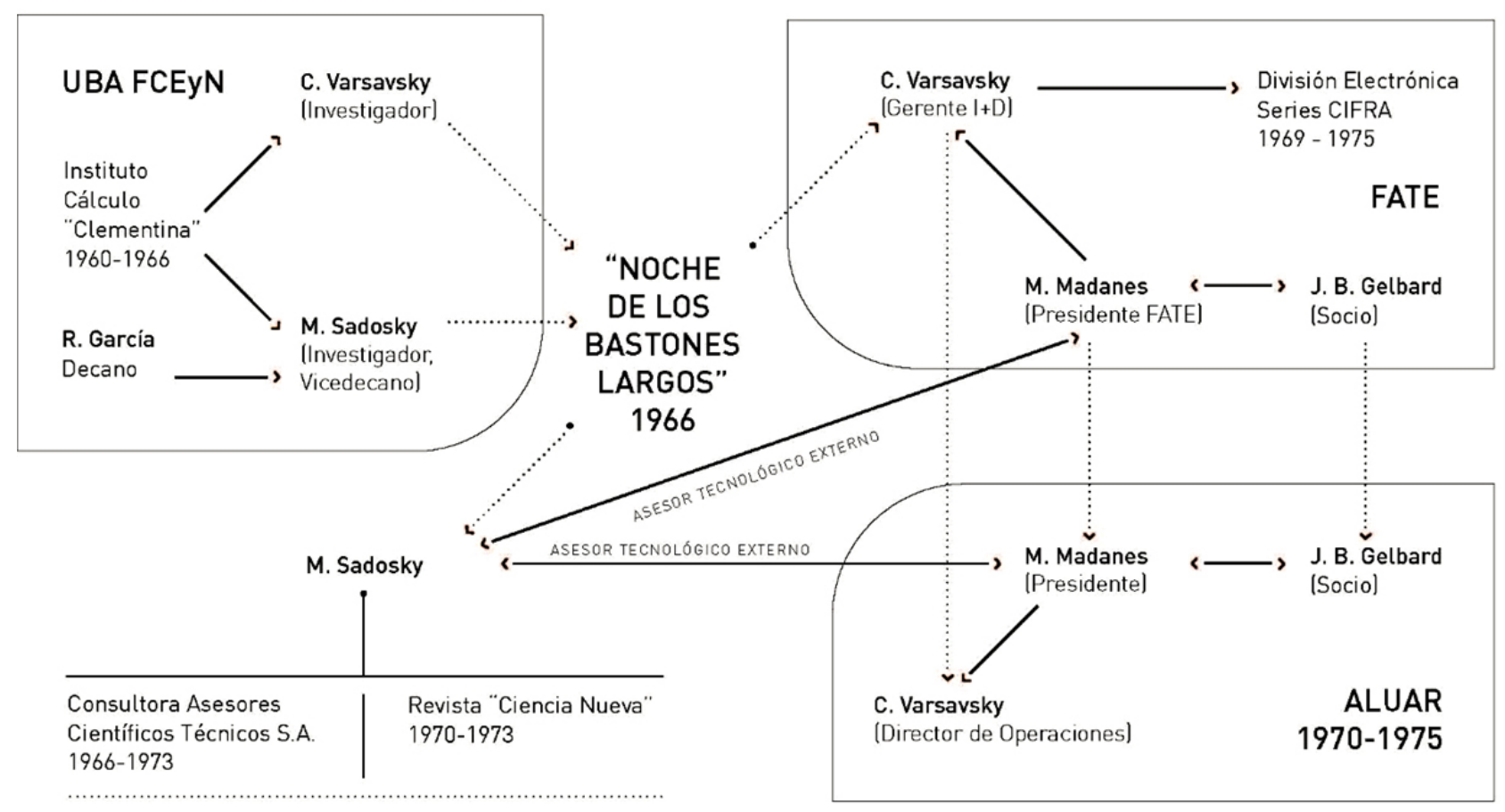

PROYECTOS INDEPENDIENTES

Figura 1. Red de actores clave del proyecto ALUAR entre 1970 y 1976. Fuente: Elaboración propia en base a De Alto (2013). 
aluminio primario tuvo su origen en el marco de un plan nacional de desarrollo industrial, los primeros acuerdos y la orientación de las definiciones iniciales estuvieron influenciados por diferentes actores claves, entre los que cabe destacar a Manuel Madanes, Manuel Sadosky, Carlos Varsavsky y José Ber Gelbard (Figura 1). Madanes, el dueño de ALUAR, tenía el convencimiento de que "el dinero estaba en innovar y resolver cosas" (Daroqui ${ }^{2}$, entrevista del 10 de mayo de 2015), idea reflejada en todo aquello que emprendía (ya desde el desarrollo de FATE y sus proyectos tecnológicos). De Alto (2013) describe a Madanes como un empresario que comprendía con claridad que la competitividad de las empresas radicaba en sus capacidades tecnológicas. Por su parte, al analizar la elección de la tecnología Montecatini (ver próxima sección), Rougier (2011) señala la obsesión de Madanes por tener bajo su control las decisiones relativas al devenir de la empresa. Su negación a incorporar la tecnología Kaiser en el inicio, a pesar de ser la recomendada por el Gobierno Nacional, fue coherente con su postura de rehusarse a contraer obligaciones y compromisos futuros que limitasen su poder.

De entrevistas a ex-empleados de la Gerencia de Investigación y Desarrollo (I+D) surge la coincidencia de reconocer que el estilo gerencial de Madanes era del tipo paternalista. La relación con “Don Manuel” y "Doña Matilde", su esposa, era cercana y ambos conocían profundamente la realidad de sus empleados. Asimismo, Madanes era quien tomaba las decisiones sobre las estrategias fundamentales a implementar en la firma, involucrándose en el inicio de cada nuevo proceso y operando sobre la comunidad de
ALUAR de manera de asegurarse su éxito.

Según De Alto (2013), a partir de la relación previa entre Sadosky y Madanes, de sus años de estudiantes de ingeniería, se inicia el vínculo entre FATE y Carlos Varsavsky. Este último, físico y astrónomo de profesión, trabajó junto a Sadosky en la Universidad de Buenos Aires (UBA), en el Instituto del Cálculo entre 1960 y 1966, y ambos habían sido desplazados de la UBA en 1966 luego de la "noche de los bastones largos".

Sadosky, quien se desempeñó como Vicedecano de la Facultad de Ciencias Exactas y Naturales de la UBA hasta el día del trágico suceso (junto al Decano Rolando García), tenía la preocupación por mantener los grupos de investigación y retener a los profesionales altamente formados de la UBA. Así lo relata Baggio, ex-jefe del Departamento de Laboratorio de ALUAR:

En el año 66' estaba terminando mi doctorado en la UBA y en la noche de los bastones largos renuncio con todo el grupo de investigación y nos vamos a Venezuela con la idea de evitar que los grupos se dispersen, ya que se pensaba que el gobierno de Onganía duraría poco y que luego podríamos volver y continuar las investigaciones. Hubo grupos que se fueron a Chile, a otros les tocó Perú y a nosotros Venezuela. Eso lo organizaron Rolando García y Manuel Sadosky (Baggio, entrevista del 10 de noviembre de 2015).

A su vez, para mantener activos a otros investigadores que se quedaron en Argentina, Sadosky fundó dos emprendimientos independientes: Ciencia Nueva, revista de ciencia y tecnología; y Asesores científico-técnicos S.A., consultora

\footnotetext{
${ }^{2}$ Físico incorporado al equipo de investigadores en 1976 y jubilado en 2014. Fue el último miembro con perfil de investigador del equipo de la Gerencia de I+D de ALUAR.
} 
especializada en computación y matemática aplicada (Figura 1). Desde allí Madanes recibía el asesoramiento de Sadosky, que en este marco le aconsejó la contratación de Varsavsky.

Desde la Gerencia de I+D de FATE, Varsavsky avanzó en dos diversificaciones de la firma. Una fue la División Electrónica de FATE en 1969, a partir del interés de Madanes en desarrollar un producto basado en una tecnología sofisticada, a lo que Varsavsky postuló como conveniente la electrónica. Esto dio lugar al desarrollo de las calculadoras CIFRA, llegando a liderar el mercado argentino y latinoamericano. La otra diversificación que emprendió Varsavsky fue el proyecto de ALUAR en 1970, una nueva empresa creada ad-hoc para participar en la producción de aluminio primario.

José Ber Gelbard, socio de Madanes, cumplió también un rol fundamental en la etapa inicial del proyecto, dada su estrecha vinculación con referentes de la política nacional, generada a partir de su liderazgo en la Confederación General Económica (CGE). Desde la década del 50', Gelbard ocupó un lugar prominente entre las figuras públicas del empresariado local y mantenía vínculos con numerosas instituciones y funcionarios de diferentes gobiernos (Rougier, 2011).

\section{La estrategia tecnológica pragmática de Varsavsky}

La tecnología utilizada en la producción de aluminio primario puede caracterizarse como madura. Varsavsky, como primer Director de Operaciones de ALUAR (lo que en la estructura actual es similar al Director Industrial), fue el responsable de definir el socio tecnológico extranjero que propondría la firma para la puesta en marcha del convenio con el Estado Nacional. Tal convenio aprobaba la instalación de la planta llave en mano con tecnología extranjera, lo que reducía una de las barreras de ingreso (Rougier, 2011). Esta medida fue un mecanismo que junto a otros (garantías de ingresos y de costos de financiación) achicaban el riesgo industrial, tecnológico y económico de la instalación y puesta en marcha de plantas de gran envergadura en el país. Si bien la política industrial desarrollista vigente durante la gestación del proyecto intentó sortear la compra de tecnología extranjera, las bases del llamado a licitación, previo al mencionado contrato, establecían requisitos que no podían cumplirse con las capacidades tecnológicas del país.

Entre los requisitos de licitación, el Estado Nacional obligaba a las empresas que se presentasen a venir con una tecnología probada, explícita (...) y en este caso particular, el Estado ha impuesto a los que quieran presentar propuestas para instalar y explotar la planta de aluminio que se presenten demostrando su capacidad previa de poder hacer aluminio. (...) De todo lo que pude averiguar en el mundo, y a pesar de que se ha dicho muchas veces de que se puede encontrar algún argentino que se haya dedicado a cualquier cosa, no encontré a ningún argentino que se haya dedicado a la fabricación de aluminio en el mundo entero (Carlos Varsavsky, 1972, p. 2).

A su vez, los plazos que establecía el concurso resultaron un obstáculo adicional a la posibilidad de desarrollar la tecnología localmente, ya que el equipo básico del proceso de fabricación de aluminio, la cuba electrolítica, requiere de pruebas de rendimiento durante un largo período. "La inteligencia argentina estaba impedida de actuar 
por la premura con que era necesario hacer la presentación" (Carlos Varsavsky, 1972, p. 2).

Es así que la propuesta pragmática que diseñó Varsavsky, con la que se presentó a licitación, consistía en un programa que combinaba adquisición de tecnología extranjera llave en mano con desarrollo propio local. En este sentido, el acuerdo de compra no incluía compromisos futuros de ALUAR con la empresa proveedora del know-how (como regalías por tonelada producida) que impusieran restricciones a las decisiones futuras de inversiones tecnológicas de la firma. Implícitamente, estos términos obligaban a ALUAR a encarar un programa tecnológico partiendo de la disponibilidad de una cuba pero con la obligación de mantenerla actualizada. "Está en manos de ALUAR dentro de diez años tener una tecnología obsoleta o dentro de diez años, con su propio esfuerzo, tener todavía la mejor tecnología del momento" (Carlos Varsavsky, 1972, p. 3).

La compra se realizó a un precio fijo a Montecatini Edison, empresa italiana que recientemente había lanzado al mercado una cuba electrolítica de 150 mil amperios de intensidad de corriente. Zavatti, Jefe de Departamento de Gestión Ambiental hasta el 2017, se refiere a la visión de Varsavsky respecto al proveedor italiano:

Varsavsky decide comprarles reconociendo que la tecnología no era la mejor del mundo pero que ofrecía posibilidades de mejora. Esto fue una visión, porque de hecho las cubas que actualmente están en marcha son basadas en las originales. Hoy tenemos equipos que son en dimensiones los mismos de 1970 (Zavatti, entrevista del 20 de septiembre de 2015).

También el ex-investigador Daroqui describe el desafío que la firma había propuesto al equipo de la Gerencia de I+D:

Lo que se compra en tecnología para ALUAR a los italianos era como un esquema de la tecnología, tal como aparecería en un libro. Por eso, los primeros años se le pone muchísima cabeza para mejorar el rendimiento. Las cubas tenían una eficiencia del $83 \%$ y en poco tiempo pasaron al 93\%. Ganaron 10 puntos en eficiencia, lo que es muchísimo dinero de ganancia. Esto fue posible porque la gente le ponía cabeza a eso y se hicieron considerables esfuerzos para mejorar la tecnología, el ambiente, el rendimiento y los materiales. Había un grupo de investigación y desarrollo especialmente dedicado a pensar en las cubas (Daroqui).

El convenio de transferencia de tecnología celebrado con la firma italiana incluyó tanto el montaje de la planta como el entrenamiento de un número importante de operarios, especialmente del área de electrólisis (Bisang, 1994). Lo interesante de la estrategia que planteó Varsavsky radica en la combinación de compra de tecnología extranjera con un plan de desarrollo propio que sortearía las limitaciones estructurales locales de ese momento. Tal propuesta permitió cumplir con las bases del llamado del Gobierno Nacional y, a la vez, generar capacidades tecnológicas endógenas. Asimismo, estas primeras definiciones fueron claves para justificar la decisión de Varsavsky de incorporar en el organigrama de la empresa una gerencia de área (dependiendo del Gerente General) dedicada exclusivamente a estudiar el proceso productivo y las mejoras incrementales a incorporar a la tecnología extranjera.

Estas definiciones se condicen con varios de los preceptos del PLACTED, particularmente 
con la visión pragmática de Sábato respecto al desarrollo tecnológico y la necesidad de crear autonomía decisional tecnológica propia. Según Dagnino et al. (1996, p. 21), para Sábato:

la condición de dominio sobre la tecnología era dada por el grado de intervención en la configuración del 'mix tecnológico' más adecuado a las condiciones locales. La necesidad de acumulación de saber tecnológico debería ser satisfecha a través de la optimización de los criterios de selección de tecnologías. (...) La soberanía nacional estaría dada por la integración del desarrollo tecnológico así generado en un proyecto nacional determinado, en primera instancia, en el plano político. No se trataba, es necesario aclarar, de seleccionar siempre las tecnologías más 'avanzadas' o las best practices que se presentaban en el estado del arte internacional, sino aquellas tecnologías que respondieran al mayor grado de adecuación a la estrategia de desarrollo. La construcción de una capacidad científica local debía ser diseñada en función de la capacidad local de producción del mix.

\section{Desafíos técnicos iniciales y primera estructura de I+D}

Según Bisang (1994), la tecnología seleccionada respondió a los estándares de la década del 60', cuando se diseñó el proyecto. Sin embargo, cuando la planta comenzó a producir en 1974 surgieron problemas técnicos y deficiencias productivas que complejizaron el desafío de alcanzar el estado del arte de la tecnología. El retraso más evidente en comparación con los pa- rámetros internacionales residía en la operación de cubas abiertas con sistema de carga lateral, ya que el modelo de Montecatini acarreaba deficiencias en el desempeño, la productividad y el impacto ambiental. Respecto a esto último, las cubas abiertas impedían la recuperación de los humos desprendidos en el proceso de electrólisis, lo que imposibilitaba su correcto control. Además, dado que la recarga manual de alúmina se realizaba cuando la cuba llegaba al efecto anódico ${ }^{3}$, esto generaba gases efecto invernadero.

Durante los primeros años de funcionamiento se dedicó mucho esfuerzo a investigar estos temas con fuerte impacto ambiental, tarea que recayó en la Jefatura de Electrólisis, dentro de la Gerencia de Investigación (Figura 2).

En la Gerencia de Investigación había muchos proyectos dedicados al entendimiento del proceso electroquímico de la cuba. Habian desarrollado cubas miniaturas que eran un hornito en donde se podían hacer procesos a nivel laboratorio que movían medio kilo de material. Conocer los parámetros básicos del manejo de una cuba eran cosas que se hacian en el laboratorio. Después, la cuba es otra historia, pero la parte fundamental estaba estudiada allí (Daroqui).

Los investigadores de la firma se enfrentaron al inicio con el desafío de contrarrestar la pronta obsolescencia de la tecnología Montecatini. La misión que Varsavsky años antes había definido para el área, respecto de trabajar en la modernización de la tecnología adquirida, se debió implementar desde el primer momento. El cerramiento de cubas fue el proyecto más impor-

${ }^{3}$ Efecto generado cuando se terminaba la carga de alúmina e iniciaba un proceso de oxidorreducción de otros elementos presentes en la cuba. 
tante con el que se involucró el equipo de investigación durante este período, por estar muy asociado a la mejora de la eficiencia productiva y al cuidado del medioambiente. A la vez resultó ser muy complejo, porque debían cerrar la brecha respecto a la tecnología internacional de la época sin dejar de operar la planta.

En lo referido al reclutamiento de los profesionales, especialmente los de I+D, el proceso estuvo muy atravesado por los hechos trágicos de la historia argentina. Los sucesos de 1966 durante el gobierno militar de Onganía contra el sector científico y, particularmente, contra la UBA llevaron a que investigadores (algunos exiliados) considerasen a ALUAR como un proyecto que renovaba sus esperanzas de desarrollar su profesión en el país. La situación del sector universitario hacia mediados de los 70' también contribuyó a atraer recursos humanos altamente formados en disciplinas básicas e ingenieriles, que en otra situación difícilmente hubiesen pensado la radicación en la Patagonia. Así relata Baggio su experiencia personal:

En Venezuela estuve casi 4 años, después el grupo se fue disolviendo y volví. Gané un concurso de profesor asociado en la Facultad de Farmacia y Bioquímica de la UBA. Ahíestuve 4 años y en el 74', después que muere Perón, asume como Rector Ottalagano, del nacionalismo católico, quien decreta que los concursos dejan de tener validez. Me lo encuentro a Bonfiglioli y me comenta que estaba en ALUAR, porque se había ido de la CONEA, y ellos estaban buscando a alguien que tuviese experiencia en trabajar con equipos de rayos $X$ en nivel de investigación y desarrollo.

En este contexto, Madanes demostró su capacidad para aprovechar la disponibilidad de profesionales formados en ciencias básicas e ingenieriles, y generar en ellos una vocación para desarrollar conocimientos vinculados a los procesos productivos de sus empresas (Harriague et al., 2007). A su vez, el asesoramiento de Varsavsky, tanto en la identificación de potenciales profesionales como en la conformación de este staff especializado, resultó ser un factor indispensable.

Ares et al. (1993) sostienen que la estrategia de incorporar tecnología en forma sistemática y, a su vez, diseñar componentes tecnológicos intermedios que pudiesen ser desarrollados localmente potenció aún más la (ya elevada) capacidad técnica y profesional. Es decir, que las definiciones posteriores relacionadas con el cambio técnico dentro de la firma generaron incluso una mayor capacidad de la Gerencia de I+D. Dicha área inició su actividad con un equipo de unos 50 profesionales provenientes de diferentes instituciones del país, un peso inusual para una firma de 300 empleados (Bisang, 1994; Harriague et al., 2007).

La primera estructura organizacional de ALUAR (Figura 2) presentaba un alto grado de profesionalización con múltiples jefaturas a cargo de doctores, algo poco habitual también en las empresas argentinas. Resulta interesante destacar el desbalance de la Gerencia de I+D, donde el mayor peso de la Gerencia de Investigación imprimía al diseño original del proyecto de ALUAR un perfil más científico, explicado en la necesidad de generar conocimientos en un país sin antecedentes en la producción de aluminio.

Muchas de estas definiciones guardan relación con nociones centrales del PLACTED, especialmente en su oposición al modelo lineal ofertista. Como señalan Galante y Lugones (2005, p. 


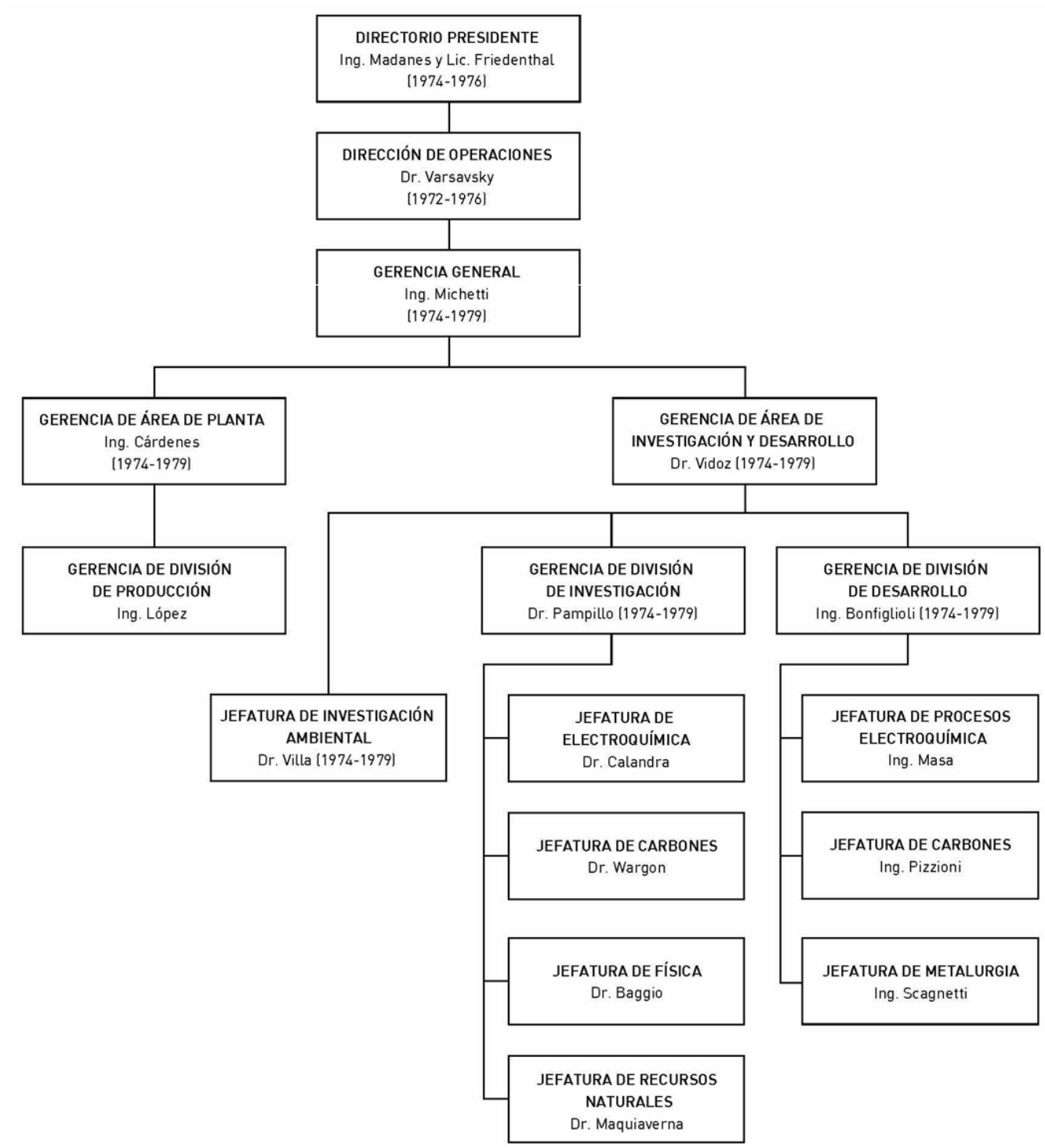

Figura 2. Organigrama parcial de ALUAR con estructura completa de Gerencia de Área de I+D (1974-1976). Fuente: Elaboración propia en base a entrevistas.

8), este movimiento:

hace hincapié en la necesidad de partir de la demanda de desarrollos tecnológicos (los cuales, en algunos casos, requieren el desarrollo de investigación básica). Es decir, a partir de la demanda del sector productivo generar o adaptar el conocimiento necesario, por lo tanto la investigación científico-tecnológica tiene como uno de sus objetivos la respuesta a la demanda de tecnología.

\section{Conclusiones}

A partir del golpe militar de 1976, la ISI y el modelo desarrollista fueron reemplazados por el modelo financiero y de ajuste estructural (Schorr, 2011), cambiando profundamente las orientaciones macroeconómicas y de la actividad industrial. A su vez, de la mano del terrorismo de Estado el país entró en una de las etapas más oscuras de su historia. En este marco se da el alejamiento de Varsavsky de ALUAR y luego su exilio en los Estados Unidos. Hacia 1977 la estructura de I+D em- 
| Ciencia, Tecnología y Política | Año 4 | No7 | e062 | Noviembre 2021 | ISSN 2618-3188 | www.revistas.un|p.edu.ar/CTyP |

pieza a simplificarse, coincidiendo con el retiro de Varsavsky y con la resolución de un conflicto sucedido durante los primeros años entre la Gerencia de Planta (a cargo del Ingeniero Cárdenes) y la de I+D (Doctor Vidoz), en disputa por el lugar de liderazgo dentro de la empresa. Finalmente, Madanes definió que la Gerencia de Planta sería asistida por la de I+D, ocupando ésta un rol de asistencia técnica y asesoramiento para las áreas de producción, del cual nunca salió. Tales cuestiones llevaron a la primera reducción del área de $1+D$, pasando de 50 a 30 miembros en 1977 y fusionando la Gerencia de División de Investigación y la de Desarrollo en una sola Gerencia de I+D, subordinada a la Gerencia de Área de Planta.

Otro factor de fuerte incidencia en el achicamiento del área de I+D fue la llegada en 1976 del Ingeniero Bargagna al cargo de Director de Operaciones, luego del alejamiento de Varsavsky. Madanes siempre demostró una capacidad para lograr acuerdos políticos beneficiosos para su holding empresarial y el cambio del Director de Operaciones encuentra sentido en la búsqueda de consensos con el gobierno militar. Massare (2014) sostiene que Bargagna mantenía diálogo con los militares, lo que resultaba estratégico. Sin embargo, no tenía una postura favorable al desarrollo de tecnología, lo que provocó las renuncias de Vidoz al cargo de Gerente de I+D y de Bonfiglioli como Gerente de División de Desarrollo.

Aún cuando Bargagna discontinuó proyectos vinculados a la I+D interna y quitó impulso a las acciones de investigación, no logró eliminar totalmente el plan original de Varsavsky. Las características del proceso de fabricación de aluminio primario, de rendimientos a escala y tecnología madura, siguieron exigiendo esfuerzos de mejoras incrementales a cargo de los profesionales de la firma, que madurarían en la década del 80' (algunos a partir de conocimientos y capacidades adquiridas durante los primeros años). Así lo describe Menegoz, Gerente de Proyectos entre 1986 y 2004 :

Fue todo un desarrollo tecnológico que se dio a partir de atacar los problemas de a uno con recursos limitados y con capacidades nuestras, porque no había a quién pedir ayuda. Montecatini, luego de vendernos las cubas, cierra a los pocos años y no había otras plantas similares a las de ALUAR. (...) Por eso digo que en la planta hay cubas $P$ chiney, que son las de la series C y D [ampliaciones puestas en marcha en 1999 y 2007], y hay cubas ALUAR, que son las de las series $A$ y $B$, ya que de Montecatini no les queda nada (Menegoz, entrevista del 19 de junio de 2018).

En definitiva, de la mano de Varsavsky ALUAR desplegó en sus primeros años una estrategia o estilo tecnológico centrado en esfuerzos e inversiones en investigación, diseño e ingeniería. Esta propuesta tuvo además como eje el desarrollo endógeno de sucesivas mejoras incrementales aplicadas a la optimización del proceso productivo, a fin de achicar el gap existente entre la tecnología italiana adquirida al inicio del proyecto y el estado del arte con el que se enfrentó la firma cuando comenzó a atender mercados externos. El éxito de la estrategia de ALUAR, en parte encuentra explicación por la existencia de conocimiento de base y recursos humanos altamente formados, que fueron capaces de especializarse rápidamente en la industria del aluminio primario. Los contactos de Varsavsky con el sistema científico y universitario nacional favorecieron la incorporación de profesionales muy calificados en la firma, que 
cubrieron cargos dentro de la Gerencia de I+D y colaboraron con la construcción de una valiosa red durante los primeros años.

ALUAR es un ejemplo de experiencias en las que las capacidades científicas lograron trasladarse y transformarse en capacidades de gestión de la tecnología, aún cuando en Argentina "estos caminos han sido sistemáticamente frustrados 0 han convivido muchas veces en tensión, o bien con políticas contrarias a estos desarrollos" (Red PLACTS, p. 5). La estrategia de Varsavsky en los inicios de ALUAR puso en práctica varios de los preceptos del pensamiento de la época, mostrando criterios propios (y estratégicos) de decisión y selección tecnológica, optando por la adquisición de una tecnología que permitiría generar un sendero de desarrollo endógeno, a partir de investigación aplicada a la resolución de problemas y de innovaciones incrementales para adecuar o mantener actualizada dicha tecnología.

\section{Bibliografía}

Ares, J., Calandra, A.; Cobo, O. (1993). Empresas líderes en desarrollo, aplicación y difusión de tecnologías ambientalmente racionales en América Latina: el caso de ALUAR ALUMINIO ARGENTINA SAIC. Buenos Aires: CEPAL.

Bisang R. (1994). ALUAR: Trayectoria económica y competitividad internacional. Cuaderno de Gestao Tecnologica No. 16, CYTED, Universidad de Sao Paulo.

Dagnino, R., Thomas, H. y Davyt, A. (1996). El pensamiento en ciencia, tecnología y sociedad en Latinoamérica: una interpretación política de su trayectoria. REDES, 7(3), 13-51.

De Alto, B. (2012). Oscar Varsavsky. Blog Relatos e Identidad. Disponible en: http://relatoseidentidad. blogspot.com/2012/02/oscar-varsavsky.html
De Alto, B. (2013). Autonomía tecnológica. La audacia de la División Electrónica de Fate. Buenos Aires: CICCUS.

Galante, O.; Lugones, A. (2005). La escuela latinoamericana de pensamiento en ciencia, tecnología y desarrollo. Revista Ciências Administrativas, 11(1), 7-17.

Galante, O.; Marí, C. (2020). Jorge Sabato y el Pensamiento Latinoamericano en Ciencia, Tecnología, Desarrollo y Dependencia. Ciencia, Tecnología y Política, 3(5), 048. https://doi.org/10.24215/26183188e048

Harriague, S.; Quilici, D.; Sbaffoni, M. (2007). El modelo del Triángulo de Sábato y tres casos argentinos. XII Seminario Latino-Americano de Gestión Tecnológica. Asociación Latino Iberoamericana de Gestión Tecnológica.

Massare, B. (2014). De los neumáticos a los chips: el rol de la I+D en el desarrollo de las calculadoras y computadoras en la División Electrónica FATE (1969-1982). III Simposio de Historia de la Informática de América Latina y el Caribe. Universidad de la República, Uruguay.

Red PLACTS. (2020). Otro estilo científico y tecnológico es posible. Ciencia, Tecnología y Política, 3(5), 050. https://doi.or$\mathrm{g} / 10.24215 / 26183188 \mathrm{e} 050$

Rougier, M. (2011). Estado y empresarios de la industria del aluminio en la Argentina: el caso ALUAR. Bernal: Ed. UNQ.

Sábato, J. (1972). Quince años de metalurgia en la Comisión Nacional de Energía Atómica. Ciencia Nueva, 15, 7-15.

Schorr, M. (2011). La desindustrialización como eje del proyecto refundacional de la economía y la sociedad en Argentina, 1976-1983. Revista América Latina de Historia Económica, 19(3), 31- 
56.

Periferia.

Varsasvky, C. (1972). Exposición de Carlos Manuel Varsasvky. Seminario de Ciencia y Tecnología Argentinas en la Industria. Programa de Transferencia. Fundación Bariloche.

Varsavsky, O. (1972). Hacia una política científica nacional. Buenos Aires: Ediciones Periferia.

Varsavsky, O. (1974). Estilos Tecnológicos. Propuestas para la selección de tecnologías bajo racionalidad socialista. Buenos Aires: Ediciones 